\title{
A case of systemic nocardiosis in systemic vasculitis and a review of the literature
}

\author{
Dinesh $\underline{\text { Sirisena }}^{1}$, MFSEM, Laith $\underline{\text { Al Swedan }}{ }^{2}$, MBBS, David Jayne ${ }^{3}$, FRCP, Kuntal Chakravarty ${ }^{2,4}$, FRCP
}

\begin{abstract}
Systemic nocardiosis is rarely reported in patients with systemic vasculitis, and is mostly described in patients experiencing long-term immunosuppression following organ transplantation. In this report, we describe a 71-year-old man who developed disseminated Nocardia infection during treatment of his underlying microscopic polyangiitis. He had no other associated risk factors. This case highlights the importance of considering rare pathogens in immunosuppressed patients who present with nonspecific symptoms, as well as the impact of such pathogens on clinical management.
\end{abstract}

Keywords: immunosuppression, microscopic polyangiitis, nocardiosis nova, systemic nocardiosis, systemic vasculitis

\section{INTRODUCTION}

Approximately 89 strains of Nocardia spp. have been identified, with 30 strains estimated to cause infections in humans. ${ }^{(1)}$ Commonly found in soil, Nocardia spp. are Grampositive aerobic bacteria with a filamentous appearance similar to fungi. They are recognised as opportunistic pathogens and can be transmitted by inhalation. Since the mid-1970s, investigators have identified patients with immunosuppression following organ transplantation and patients with acquired immunodeficiency syndrome as those who are at the greatest risk of infection. ${ }^{(2,3)}$ Nocardiosis (Norcardia infection) can also manifest in immunocompetent individuals, although this is relatively rare.

The clinical presentation of nocardiosis is primarily pulmonary, with reported identification in cases of necrotising pneumonia, empyema and lung abscesses. ${ }^{(4)}$ However, other manifestations, such as cutaneous or cerebral infection with cellulitic nodular changes and abscesses that lead to space-occupying, lesion-like presentations, have also been documented in the literature. ${ }^{(5,6)}$ The primary challenge with nocardiosis is in arriving at a diagnosis. Although well-reported in the medical literature, the relative rarity of this infection can lead to it being overlooked and misdiagnosed as other pathologies. The nonspecific presentation and imaging findings of the disease warrant culture and tissue diagnosis. A review by Sullivan and Chapman in 2009 highlighted that Nocardia is commonly mistaken for fungi, and therefore, infections may not be identified or treated appropriately, even with culture.(1)

Treatment of nocardiosis is dependent on the pathogen subtype and the antimicrobial options available. This can include quinolone, cephalosporin, penicillin and trimethoprim, among others. ${ }^{(3)}$ The variety of possible treatment options emphasises the importance of accurate typing, which is another challenge

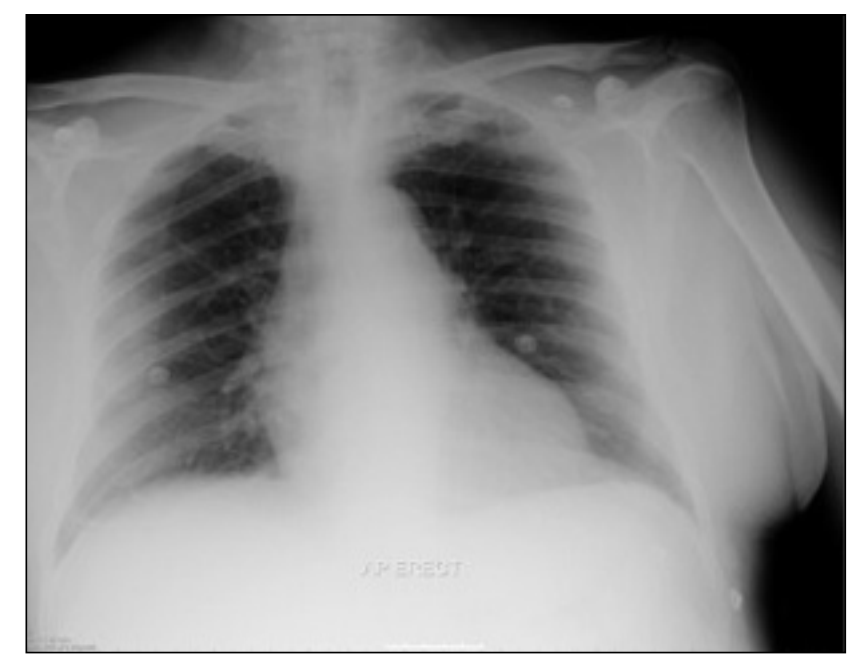

Fig. 1 Initial plain anteroposterior chest radiograph shows cardiomegaly, with dilated peripheral veins and oligaemia at the base of the lungs.

posed by this pathogen. While Norcadia infection is rarely observed in patients with vasculitis, Tabrizi ${ }^{(7)}$ has described the challenges in treating a patient with microscopic polyangiitis (MPA) and Nocardia asteroids infection with resultant pericarditis. This present case report describes systemic nocardiosis in a patient with MPA due to Nocardia nova.

\section{CASE REPORT}

A 71-year-old man with a history of psoriasis and hypertension developed progressive shortness of breath, which was preceded by increasing polyarthralgia, malaise, painful mouth ulcers and a skin rash for approximately three weeks. Initial treatment with oral augmentin, prescribed by a general practitioner, failed to improve his symptoms. The patient was admitted to the emergency department in early spring 2009. At the time of presentation, the patient was observed to have type 2 respiratory failure and renal dysfunction with haematuria and

\footnotetext{
${ }^{1}$ Department of Exercise Physiology, Hammersmith Hospital, London, ${ }^{2}$ Department of Rheumatology, Barking Havering and Redbridge University Hospital NHS Trust, Essex, ${ }^{3}$ Department of Nephrology, Addenbrooke's Hospital NHS Trust, Cambridge, ${ }^{4}$ Postgraduate Medical School, University of Bedfordshire, Bedfordshire, United Kingdom Correspondence: Dr Dinesh Sirisena, Sports and Exercise Medicine Registrar, Department of Exercise Physiology, Hammersmith Hospital, London, W12 OHS, United Kingdom. dineshsirisena@doctors.org.uk
} 


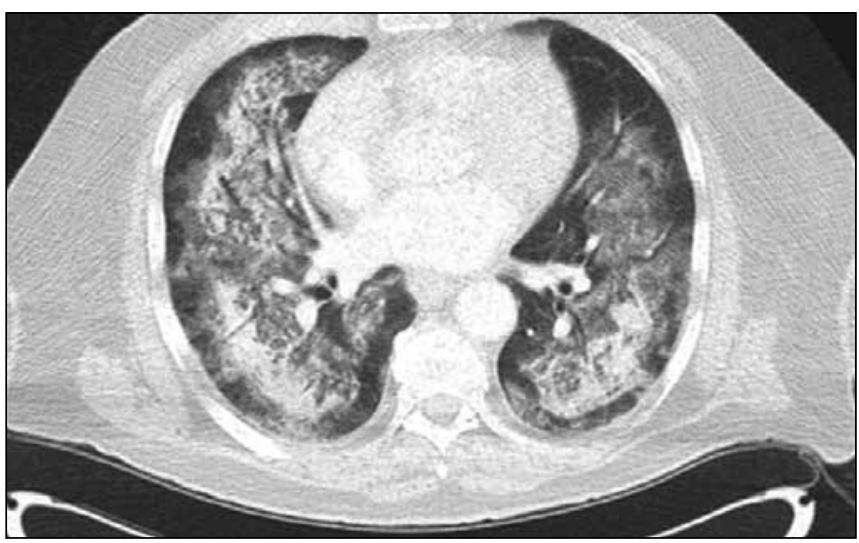

Fig. 2 Initial axial CT image of the chest shows abnormal alveolar shadowing involving both the upper-and mid-zones, with sparing of the lung bases, suggestive of acute pneumonitis.
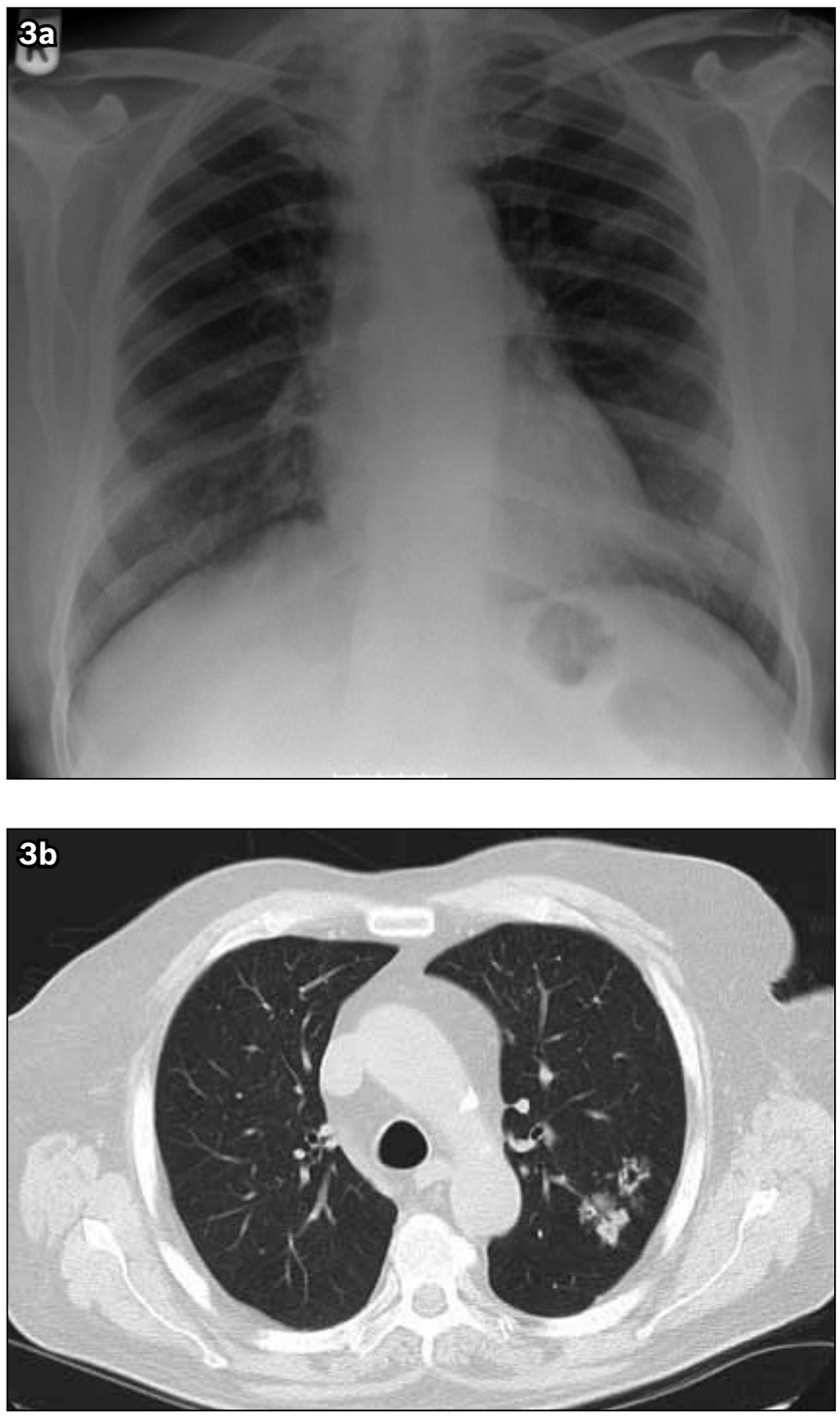

Fig. 3 (a) Plain anteroposterior chest radiograph shows a left midzone cavitating lesion, which led to the suspicion of tuberculosis and subsequent bronchoalveolar lavage. (b) Axial non-contrast CT image of the chest following plain chest radiography shows the cavitating lesion in the left mid-zone.

proteinuria. He was not on any disease-modifying drugs such as methotrexate, but was taking a nonsteroidal anti-inflammatory drug (diclofenac) for his joint pain symptoms.
Table I. Results of the patient's blood tests, including full blood count, test for inflammatory markers and serology.

\begin{tabular}{lcc}
\hline Variable & Patient's results & Normal range \\
\hline Haemoglobin (g/dL) & 9.7 & $13-18$ \\
White blood cells (/L) & $9.9 \times 10^{9}$ & $4-11 \times 10^{9}$ \\
Neutrophils (/L) & $7.6 \times 10^{9}$ & $2-7.5 \times 10^{9}$ \\
Platelets (/L) & $188 \times 10^{9}$ & $150-400 \times 10^{9}$ \\
Eosinophils (/L) & $0.3 \times 10^{9}$ & $0.04-0.44 \times 10^{9}$ \\
ESR (mm/h) & 44 & $<5$ \\
Creatinine (mmol/L) & 182 & $60-120$ \\
Urea (mmol/L) & 13.5 & $2.5-7.5$ \\
ALT (IU/L) & 43 & $10-50$ \\
Albumin (g/L) & 34 & $35-50$ \\
pANCA & Positive & Myeloperoxidase \\
Anti-GBM & & $>100 \mathrm{U} / \mathrm{mL}$ \\
\hline
\end{tabular}

ALT: alanine transaminase; pANCA: perinuclear anti-neutrophil cytoplasmic antibodies; ESR: erythrocyte sedimentation rate; GBM: glomerular basement membrane; MPO: myeloperoxidase.

Microbiological investigations, including blood culture, viral serology (for hepatitis A, B and C, cytomegalovirus $[\mathrm{CMV}]$, human immunodeficiency virus [HIV] and parvovirus), as well as throat and nasal swabs for methicillin-resistant Staphylococcus aureus, were all negative. Chest radiography revealed cardiomegaly with dilated peripheral veins and oligaemia in the lung bases (Fig. 1). Echocardiogram showed mild left ventricular hypertrophy with good left ventricular function, while computed tomography (CT) of the chest showed abnormal alveolar shadowing involving the upper- and mid-zones, with sparing of the lung bases, suggestive of acute pneumonitis (Fig. 2). A pulmonary-renal syndrome such as Goodpasture's syndrome was suspected, and renal biopsy confirmed renal vasculitis with crescent formation.

The patient was started on intravenous methylprednisolone and cyclophosphamide at conventional doses to induce remission of his active vasculitis, followed by oral treatment as per national guidelines. ${ }^{(8)}$ Serology results showed that the patient was positive for perinuclear anti-neutrophil cytoplasmic antibodies and negative for anti-glomerular basement membrane (anti-GBM) (Table I); thus a diagnosis of MPA was made. The patient's renal function subsequently improved and there was radiological resolution of the signs, leading to his discharge from the intensive treatment unit after three weeks.

Three months after the patient's emergency admission, he relapsed with progressive shortness of breath and a productive cough. Chest radiography and CT revealed previously unseen cavitating lesions in the left mid-zone of the chest (Fig. 3). Bronchoalveolar lavage was performed and the washout showed high titres of CMV (216,000 copies/mL), which was associated with a marginal elevation in serum CMV DNA PCR $(4,000$ copies $/ \mathrm{mL})$. By the time the CMV DNA PCR result was obtained, treatment with valgancyclovir had already commenced on the basis of clinical suspicion. As the cavitating lesions raised the suspicion of either cavitating pulmonary 
tuberculosis or possible Wegener's granulomatosis, a lung biopsy was performed. The biopsy revealed organising pneumonia with features of intra-alveolar haemorrhage and fibrous deposition consistent with MPA, as well as one suspicious CMV inclusion body.

Despite treatment with intravenous augmentin for suspected community-acquired pneumonia, the patient's health continued to deteriorate clinically, with worsening dyspnoea and occasional haemoptysis. Chest radiograph and CT performed at that time confirmed the development of further lesions, in keeping with pulmonary vasculitis. The patient responded favourably to rituximab, with improvement in his renal function within two weeks. Repeat chest radiography showed considerable improvement (Fig. 4) and the patient was discharged home with a prescribed dose of oral prednisolone (10 mg) with bone and gastric protection.

Approximately two months after discharge, the patient returned to the emergency department with an acute onset of severe buttock pain. A clinical diagnosis of an abscess related to a possible staphylococcal infection was made. Local drainage was performed, followed by intravenous, and then oral, antibiotics (co-amoxiclav) prior to discharge from the emergency department. A few weeks later, the patient was readmitted with worsening respiratory symptoms. Microbiological culture of the pus from the patient's buttock abscess grew Gram-positive rods with a beaded appearance and positive acid-fast staining, which is suspicious for Nocardia spp. Multiple pulmonary abscesses, confirmed by $\mathrm{CT}$ of the lung, were subjected to transthoracic lung biopsy. The mode of Nocardia dissemination could not be ascertained, as blood cultures were negative. Although the patient was asymptomatic, previous experience with nocardiosis prompted magnetic resonance (MR) imaging of the brain, which showed a solitary ring lesion on the left parietotemporal lobe (Fig. 5). No rise in cerebrospinal fluid pressure was detected and no evidence of malignancy was found.

Lung biopsy confirmed infection with Nocardia nova, and treatment with intravenous imipenum and co-trimoxazole was commenced with monitoring of the patient's renal function. This regimen continued for six weeks until antibiotic sensitivity results were available, after which imipenum was replaced with meropenum. At the end of the six-week intravenous treatment, oral azithromycin and co-trimoxazole were commenced for three months, after which the patient was maintained on co-trimoxazole alone for almost a year. No relapse of Nocardia infection or any renal complication was encountered during that period. In the course of the patient's treatment, we adhered to the national guidelines on long-term use of co-trimoxazole and maintained regular renal monitoring. MR imaging of the brain, repeated 18 months after the initial $M R$ investigation, showed a near-complete absence of the lesion previously identified.

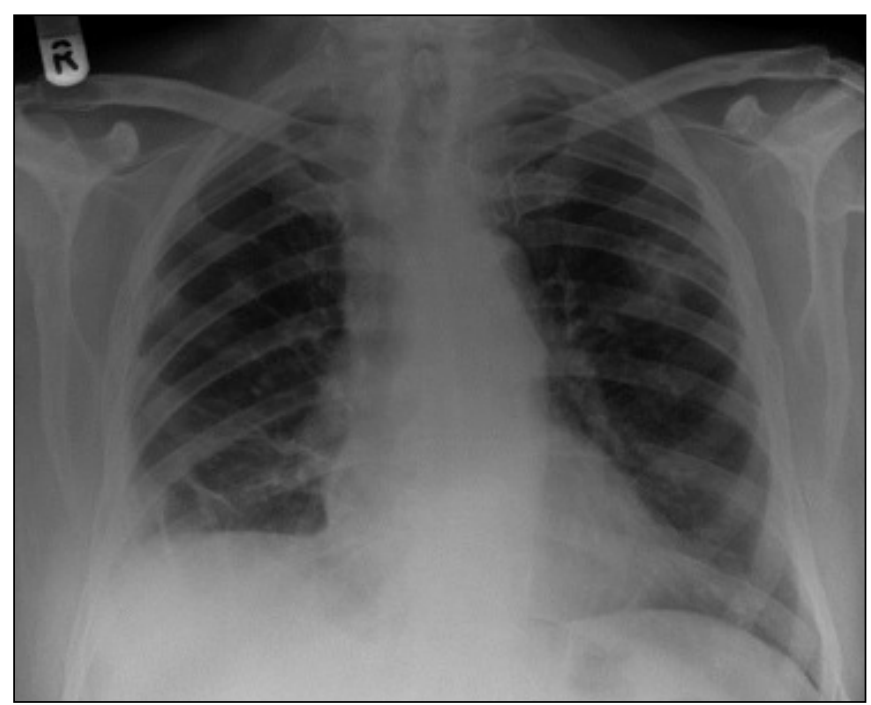

Fig. 4 Plain anteroposterior chest radiograph post-rituximab treatment shows resolution of the left mid-zone cavitating lesion.

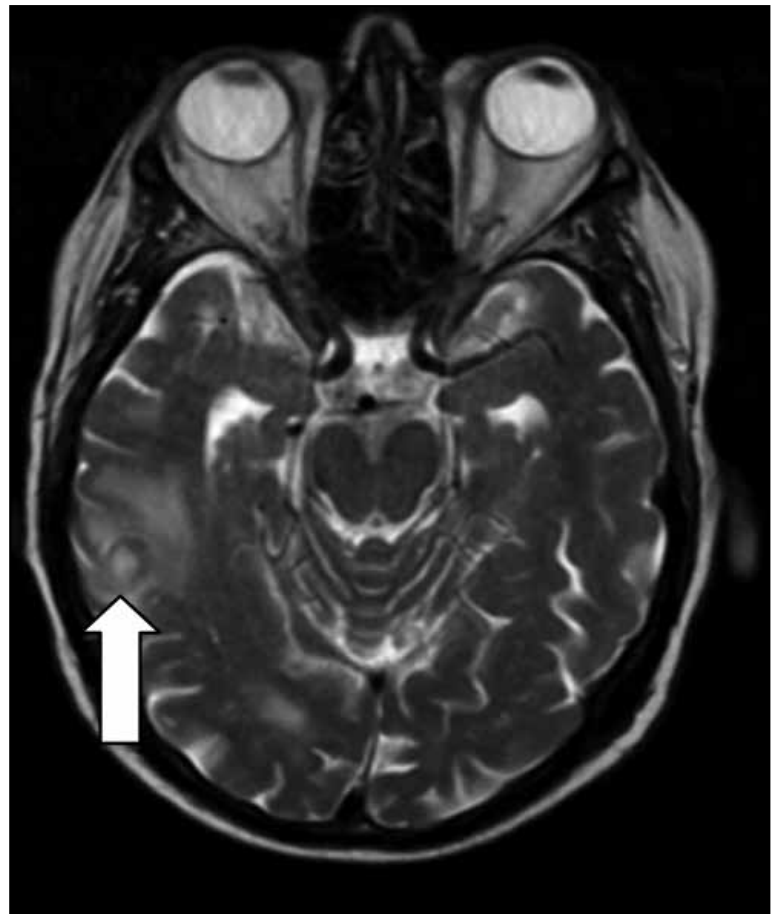

Fig. 5 T2-W transverse MR image of the brain shows a ring lesion (arrow) in the left temperoparietal region.

\section{DISCUSSION}

Disseminated nocardiosis has not been reported in patients with systemic vasculitis, especially MPA, even in large studies. Randomised European trials in vasculitis have not reported Nocardia infection in their study patients. ${ }^{(9)}$ As such, little is known about the outcome of this condition. Nocardiosis has, however, been described in patients with non-Hodgkin's lymphoma and in renal transplant patients following rituximab therapy. ${ }^{(10,11)}$ Our report describes the first case of MPA complicated by systemic nocardiosis that was successfully treated.

Nocardia spp. are ubiquitous environmental micro-organisms belonging to a diverse group of Actinomycetes. ${ }^{(12,13)}$ In the majority of cases, Nocardia infection is opportunistic, targeting 
immunocompromised patients such as those who are on long-term steroids and HIV-positive, as well as chemotherapy or post- organ transplantation patients. ${ }^{(14)}$ Patients who have undergone kidney, heart or liver transplantation are most commonly affected. ${ }^{(15)}$ Infection with CMV has also been described as a cofactor in the development of nocardiosis, as seen in our case, in which CMV performed an important immunomodulatory role in the development of nocardiosis. One matched case-control study ${ }^{(2)}$ elucidated common risk factors for nocardiosis in organ transplant patients that were almost identical to the findings in our patient who had immune suppression, due to systemic steroid therapy, and a history of CMV infection.

Our patient's presentation was complex as his initial lung biopsy suggested CMV infection. This was treated with appropriate antiviral medication. At his subsequent admission, his worsening breathlessness and repeat CT of the chest suggested granulomatous disease, raising the possibility of tuberculosis following immunosuppressive therapy. A second lung biopsy was mandatory to rule out either granulomatous infection or spread of Nocardia into the lungs. At the patient's initial presentation to the hospital, nocardiosis was not suspected as the degree of renal impairment suggested an autoimmune pathology. Also, while it has been noted that lung cavities secondary to Nocardia infection are able to self-resolve, the worsening condition in our patient made treatment necessary.

The presence of simultaneous lung and brain abscesses has been documented as a reliable indication of an underlying Nocardia infection. ${ }^{(16)}$ Although our patient was asymptomatic, the MR imaging findings were consistent with characteristics that have been noted in past studies. ${ }^{(17,18)}$ This leads to the dilemma of whether patients undergoing immunosuppressive therapy should be screened early for nocardiosis, especially if they present with nonspecific symptoms.

In our patient, nocardiosis was caused by Nocardia nova, which was sensitive to co-trimoxazole and imipenum. Six weeks of treatment with these antibiotics led to complete clinical and radiological recovery in the patient. However, the asymptomatic brain involvement proved challenging, requiring carefully selected antibiotics with high cerebrospinal fluid penetration and prolonged treatment with co-trimoxazole. Serial MR imaging of our patient's brain showed gradual improvement in the size of the intracranial lesion as treatment progressed.

In conclusion, the incidence of nocardiosis in patients with vasculitis and its prognosis with treatment remain unclear. Our case is unique on two accounts - it is the first reported case of systemic infection in MPA, and the patient experienced rapid recovery due to early detection and careful management. Most cases described in the literature had less favourable outcomes with significant mortality. It may be worthwhile to consider regular prophylactic therapy for systemic nocardiosis in patients with systemic vasculitis undergoing immunosuppressive treatment, since their immune status is similar to patients undergoing immunosuppression following organ transplantation. It may also be prudent to keep co-trimoxazole as the first-line therapeutic or prophylactic option until antibiotic sensitivity results are available to shed light on the management of the condition. Finally, this case highlights the importance of considering nocardiosis in immunocompromised patients with unusual presentations, as well as the possibility of a cure if the infection is detected early and managed carefully.

\section{REFERENCES}

1. Sullivan DC, Chapman SW. Bacteria that masquerade as fungi: actinomycosis/nocardia. Proc Am Thorac Soc 2010; 7:216-21.

2. Peleg AY, Husain S, Qureshi ZA, et al. Risk factors, clinical characteristics, and outcome of Nocardia infection in organ transplant recipients: a matched case-control study. Clin Infect Dis 2007; 44:1307-14.

3. Provost $F$, Laurent $F$, Blanc MV, Boiron P. Transmission of nocardiosis and molecular typing of Nocardia species: a short review. Eur J Epidemiol 1997; 13:235-8

4. Kanne JP, Yandow DR, Mohammed TL, Meyer CA. CT findings of pulmonary nocardiosis. AJR Am J Roentgenol 2011; 197:W266-72.

5. Dodiuk-Gad R, Cohen E, Ziv M, et al. Cutaneous nocardiosis: report of two cases and review of the literature. Int J Derm 2010; 49:1380-5.

6. Kilincer C, Hamamcioglu MK, Simsek O, et al. Nocardial brain abscess: review of clinical management. J Clin Neurosci 2006; 13:481-5.

7. Tabrizi SJ. Nocardia pericarditis. BMJ 1994; 309:1495-7.

8. Lapraik C, Watts R, Bacon P, et al. BSR and BHPR guidelines for the management of adults with ANCA associated vasculitis. Rheumatology (Oxford) 2007; 46:1615-6.

9. Yew S, Jayne D, Hoglund $P$, et al. The disease characteristics of ANCA associated vasculitis: 735 patients recruited at diagnosis to six European vasculitis study group trials (abstract). Nephrology 2012; 17(2 suppl): 45.

10. Flohr TR, Sifri CD, Brayman KL, et al. Nocardiosis in a renal transplant recipient following Rituximab preconditioning. Ups J Med Sci 2009; 114:62-4.

11. Kundranda MN, Spiro TP, Muslimani A, et al. Cerebral nocardiosis in a patient with NHL treated with rituximab. Am J Hematol 2007; 82:1033-4.

12. Brown-Elliott BA, Brown JM, Conville PS, Wallace RJ Jr. Clinical and laboratory features of Nocardia spp. based on current molecular taxonomy. Clin Microbiol Rev 2006; 19:259-82.

13. Saubolle MA, Sussland D. Nocardiosis: review of clinical and laboratory experience. J Clin Microbiol 2003; 41:4497-501.

14. Lerner PI. Nocardiosis. Clin Infect Dis 1996; 22:891-903.

15. Simpson GL, Stinson EB, Egger MJ, Remington JS. Nocardial infections in the immunocompromised host: A detailed study in a defined population. Rev Infect Dis 1981; 3:492-507.

16. Clinicopathologic conference. Headache, mental status changes and death in a 36-year-old woman with lupus. Am J Med 1989; 86:94-102.

17. Cianfoni A, Calandrelli R, De Bonis $P$, et al. Nocardia brain abscess mimicking high-grade necrotic tumor on perfusion MRI. J Clin Neurosci 2010; 17:1080-2.

18. Malincarne L, Marroni M, Farina C, et al. Primary brain abscess with Nocardia farcinica in an immunocompetent patient. Clin Neurol Neurosurg 2002; 104:132-5. 